\title{
Efficient Context and Schema Fusion Networks for Multi-Domain Dialogue State Tracking
}

\author{
Su Zhu, Jieyu Li, Lu Chen* and Kai Yu* \\ State Key Laboratory of Media Convergence Production Technology and Systems \\ MoE Key Lab of Artificial Intelligence, AI Institute, Shanghai Jiao Tong University \\ SpeechLab, Department of Computer Science and Engineering \\ Shanghai Jiao Tong University, Shanghai, China \\ \{paul2204, oracion, chenlusz,kai.yu\}esjtu.edu.cn
}

\begin{abstract}
Dialogue state tracking (DST) aims at estimating the current dialogue state given all the preceding conversation. For multi-domain DST, the data sparsity problem is a major obstacle due to increased numbers of state candidates and dialogue lengths. To encode the dialogue context efficiently, we utilize the previous dialogue state (predicted) and the current dialogue utterance as the input for DST. To consider relations among different domain-slots, the schema graph involving prior knowledge is exploited. In this paper, a novel context and schema fusion network is proposed to encode the dialogue context and schema graph by using internal and external attention mechanisms. Experiment results show that our approach can outperform strong baselines, and the previous state-of-the-art method (SOM-DST) can also be improved by our proposed schema graph.
\end{abstract}

\section{Introduction}

Dialogue state tracking (DST) is a key component in task-oriented dialogue systems which cover certain narrow domains (e.g., booking hotel and travel planning). As a kind of context-aware language understanding task, DST aims to extract user goals or intents hidden in human-machine conversation and represent them as a compact dialogue state, i.e., a set of slots and their corresponding values. For example, as illustrated in Fig. 1, (slot, value) pairs like (name, huntingdon marriott hotel) are extracted from the dialogue. It is essential to build an accurate DST for dialogue management (Young et al., 2013), where dialogue state determines the next machine action and response.

Recently, motivated by the tremendous growth of commercial dialogue systems like Apple Siri, Microsoft Cortana, Amazon Alexa, or Google Assistant, multi-domain DST becomes crucial to help

\footnotetext{
${ }^{*}$ The corresponding authors are Lu Chen and Kai Yu.
}

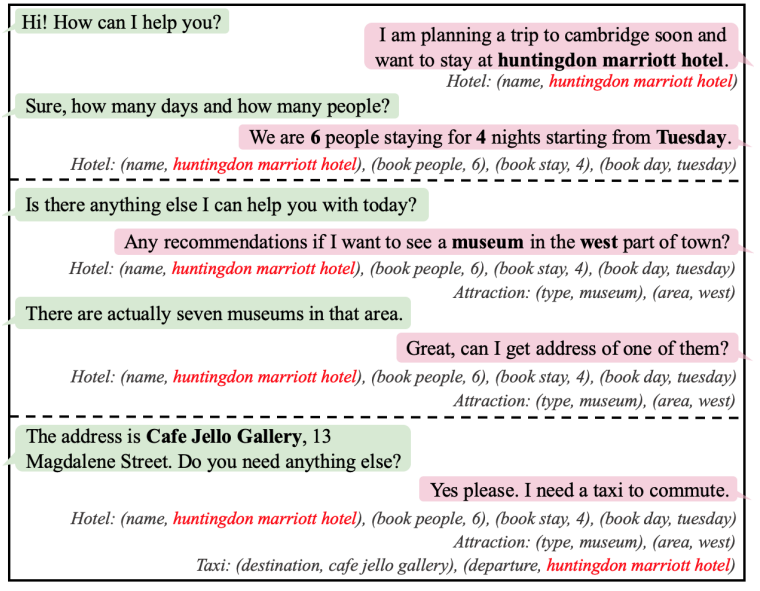

Figure 1: An example of multi-domain dialogues. Utterances at the left side are from the system agent, and utterances at the right side are from a user. The dialogue state of each domain is represented as a set of (slot, value) pairs.

users across different domains (Budzianowski et al., 2018; Eric et al., 2019). As shown in Fig. 1, the dialogue covers three domains (i.e., Hotel, Attraction and Taxi). The goal of multidomain DST is to predict the value (including NONE) for each domain-slot pair based on all the preceding dialogue utterances. However, due to increasing numbers of dialogue turns and domainslot pairs, the data sparsity problem becomes the main issue in this field.

To tackle the above problem, we emphasize that DST models should support open-vocabulary based value decoding, encode context efficiently and incorporate domain-slot relations:

1. Open-vocabulary DST is essential for realworld applications (Wu et al., 2019; Gao et al., 2019; Ren et al., 2019), since value sets for some slots can be very huge and variable (e.g., song names).

2. To encode the dialogue context efficiently, we 
attempt to get context representation from the previous (predicted) dialogue state and the current turn dialogue utterance, while not concatenating all the preceding dialogue utterances.

3. To consider relations among domains and slots, we introduce the schema graph which contains domain, slot, domain-slot nodes and their relationships. It is a kind of prior knowledge and may help alleviate the data imbalance problem.

To this end, we propose a multi-domain dialogue state tracker with context and schema fusion networks (CSFN-DST). The fusion network is exploited to jointly encode the previous dialogue state, the current turn dialogue and the schema graph by internal and external attention mechanisms. After multiple layers of attention networks, the final representation of each domain-slot node is utilized to predict the corresponding value, involving context and schema information. For the value prediction, a slot gate classifier is applied to decide whether a domain-slot is mentioned in the conversation, and then an RNN-based value decoder is exploited to generate the corresponding value.

Our proposed CSFN-DST is evaluated on MultiWOZ 2.0 and MultiWOZ 2.1 benchmarks. Ablation study on each component further reveals that both context and schema are essential. Contributions in this work are summarized as:

- To alleviate the data sparsity problem and enhance the context encoding, we propose exploiting domain-slot relations within the schema graph for open-vocabulary DST.

- To fully encode the schema graph and dialogue context, fusion networks are introduced with graph-based, internal and external attention mechanisms.

- Experimental results show that our approach surpasses strong baselines, and the previous state-of-the-art method (SOM-DST) can also be improved by our proposed schema graph.

\section{Related Work}

Traditional DST models rely on semantics extracted by natural language understanding to predict the current dialogue states (Young et al., 2013; Williams et al., 2013; Henderson et al., 2014d; Sun et al., 2014b,a; Yu et al., 2015), or jointly learn language understanding in an end-to-end way (Henderson et al., 2014b,c). These methods heavily rely on hand-crafted features and complex domain-specific lexicons for delexicalization, which are difficult to extend to new domains. Recently, most works about DST focus on encoding dialogue context with deep neural networks (such as CNN, RNN, LSTM-RNN, etc.) and predicting a value for each possible slot (Mrkšić et al., 2017; Xu and Hu, 2018; Zhong et al., 2018; Ren et al., 2018).

Multi-domain DST Most traditional state tracking approaches focus on a single domain, which extract value for each slot in the domain (Williams et al., 2013; Henderson et al., 2014a). They can be directly adapted to multi/mixed-domain conversations by replacing slots in a single domain with domain-slot pairs (i.e. domain-specific slots) (Ramadan et al., 2018; Gao et al., 2019; Wu et al., 2019; Zhang et al., 2019; Kim et al., 2019). Despite its simplicity, this approach for multi-domain DST extracts value for each domain-slot independently, which may fail to capture features from slot co-occurrences. For example, hotels with higher stars are usually more expensive (price_range).

Predefined ontology-based DST Most of the previous works assume that a predefined ontology is provided in advance, i.e., all slots and their values of each domain are known and fixed (Williams, 2012; Henderson et al., 2014a). Predefined ontology-based DST can be simplified into a value classification task for each slot (Henderson et al., 2014c; Mrkšić et al., 2017; Zhong et al., 2018; Ren et al., 2018; Ramadan et al., 2018; Lee et al., 2019). It has the advantage of access to the known candidate set of each slot, but these approaches may not be applicable in the real scenario. Since a full ontology is hard to obtain in advance (Xu and $\mathrm{Hu}$, 2018), and the number of possible slot values could be substantial and variable (e.g., song names), even if a full ontology exists (Wu et al., 2019).

Open-vocabulary DST Without a predefined ontology, some works choose to directly generate or extract values for each slot from the dialogue context, by using the encoder-decoder architecture (Wu et al., 2019) or the pointer network (Gao et al., 2019; Ren et al., 2019; Le et al., 2020). They can improve the scalability and robustness to unseen slot values, while most of them are not efficient in context encoding since they encode all the previous utterances at each dialogue turn. Notably, a multi- 
domain dialogue could involve quite a long history, e.g., MultiWOZ dataset (Budzianowski et al., 2018) contains about 13 turns per dialogue on average.

Graph Neural Network Graph Neural Network (GNN) approaches (Scarselli et al., 2009; Veličković et al., 2018) aggregate information from graph structure and encode node features, which can learn to reason and introduce structure information. Many GNN variants are proposed and also applied in various NLP tasks, such as text classification (Yao et al., 2019), machine translation (Marcheggiani et al., 2018), dialogue policy optimization (Chen et al., 2018, 2019) etc. We introduce graph-based multi-head attention and fusion networks for encoding the schema graph.

\section{Problem Formulation}

In a multi-domain dialogue state tracking problem, we assume that there are $M$ domains (e.g. taxi, hotel) involved, $\mathcal{D}=\left\{d_{1}, d_{2}, \cdots, d_{M}\right\}$. Slots included in each domain $d \in \mathcal{D}$ are denoted as a set $\mathcal{S}^{d}=\left\{s_{1}^{d}, s_{2}^{d}, \cdots, s_{\left|\mathcal{S}^{d}\right|}^{d}\right\} .{ }^{1}$ Thus, there are $J$ possible domain-slot pairs totally, $\mathcal{O}=$ $\left\{O_{1}, O_{2}, \cdots, O_{J}\right\}$, where $J=\sum_{m=1}^{M}\left|\mathcal{S}^{d_{m}}\right|$. Since different domains may contain a same slot, we denote all distinct $N$ slots as $\mathcal{S}=$ $\left\{s_{1}, s_{2}, \cdots, s_{N}\right\}$, where $N \leq J$.

A dialogue can be formally represented as $\left\{\left(A_{1}, U_{1}, B_{1}\right),\left(A_{2}, U_{2}, B_{2}\right), \cdots,\left(A_{T}, U_{T}, B_{T}\right)\right\}$, where $A_{t}$ is what the agent says at the $t$-th turn, $U_{t}$ is the user utterance at $t$ turn, and $B_{t}$ denotes the corresponding dialogue state. $A_{t}$ and $U_{t}$ are word sequences, while $B_{t}$ is a set of domain-slot-value triplets, e.g., (hotel, price_range, expensive). Value $v_{t j}$ is a word sequence for $j$-th domain-slot pair at the $t$-th turn. The goal of DST is to correctly predict the value for each domain-slot pair, given the dialogue history.

Most of the previous works choose to concatenate all words in the dialogue history, $\left[A_{1}, U_{1}, A_{2}, U_{2}, \cdots, A_{t}, U_{t}\right]$, as the input. However, this may lead to increased computation time. In this work, we propose to utilize only the current dialogue turn $A_{t}, U_{t}$ and the previous dialogue state $B_{t-1}$ to predict the new state $B_{t}$. During the training, we use the ground truth of $B_{t-1}$, while the previous predicted dialogue state would be used in the inference stage.

Schema Graph To consider relations between

\footnotetext{
${ }^{1}$ For open-vocabulary DST, possible values for each slo $s \in \mathcal{S}^{d}$ are not known in advance.
}

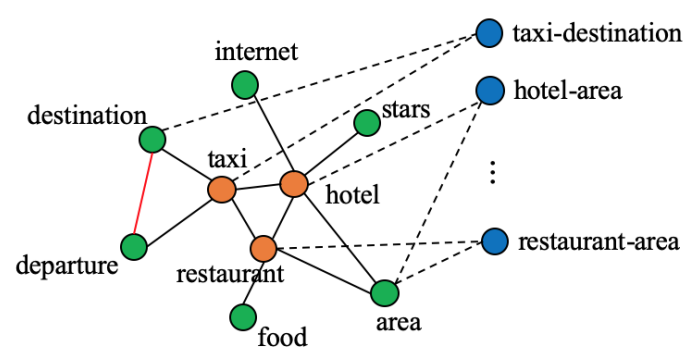

Figure 2: An example of schema graph. Domain nodes are in orange, slot nodes are in green and domain-slot nodes are in blue.

different domain-slot pairs and exploit them as an additional input to guide the context encoding, we formulate them as a schema graph $G=(V, E)$ with node set $V$ and edge set $E$. Fig. 2 shows an example of schema graph. In the graph, there are three kinds of nodes to denote all domains $\mathcal{D}$, slots $\mathcal{S}$, and domain-slot pairs $\mathcal{O}$, i.e., $V=\mathcal{D} \cup \mathcal{S} \cup \mathcal{O}$. Four types of undirected edges between different nodes are exploited to encode prior knowledge:

1. $\left(d, d^{\prime}\right)$ : Any two domain nodes, $d \in \mathcal{D}$ and $d^{\prime} \in \mathcal{D}$, are linked to each other.

2. $(s, d):$ We add an edge between slot $s \in \mathcal{S}$ and domain $d \in \mathcal{D}$ nodes if $s \in \mathcal{S}^{d}$.

3. $(d, o)$ and $(s, o)$ : If a domain-slot pair $o \in \mathcal{O}$ is composed of the domain $d \in \mathcal{D}$ and slot $s \in \mathcal{S}$, there are two edges from $d$ and $s$ to this domain-slot node respectively.

4. $\left(s, s^{\prime}\right)$ : If the candidate values of two different slots $\left(s \in \mathcal{S}\right.$ and $s^{\prime} \in \mathcal{S}$ ) would overlap, there is also an edge between them, e.g., destination and departure, leave_at and arrive_by.

\section{Context and Schema Fusion Networks for Multi-domain DST}

In this section, we will introduce our approach for multi-domain DST, which jointly encodes the current dialogue turn $\left(A_{t}\right.$ and $\left.U_{t}\right)$, the previous dialogue state $B_{t-1}$ and the schema graph $G$ by fusion networks. After that, we can obtain contextaware and schema-aware node embeddings for all $J$ domain-slot pairs. Finally, a slot-gate classifier and RNN-based value decoder are exploited to extract the value for each domain-slot pair.

The architecture of CSFN-DST is illustrated in Fig. 3, which consists of input embeddings, context schema fusion network and state prediction modules. 


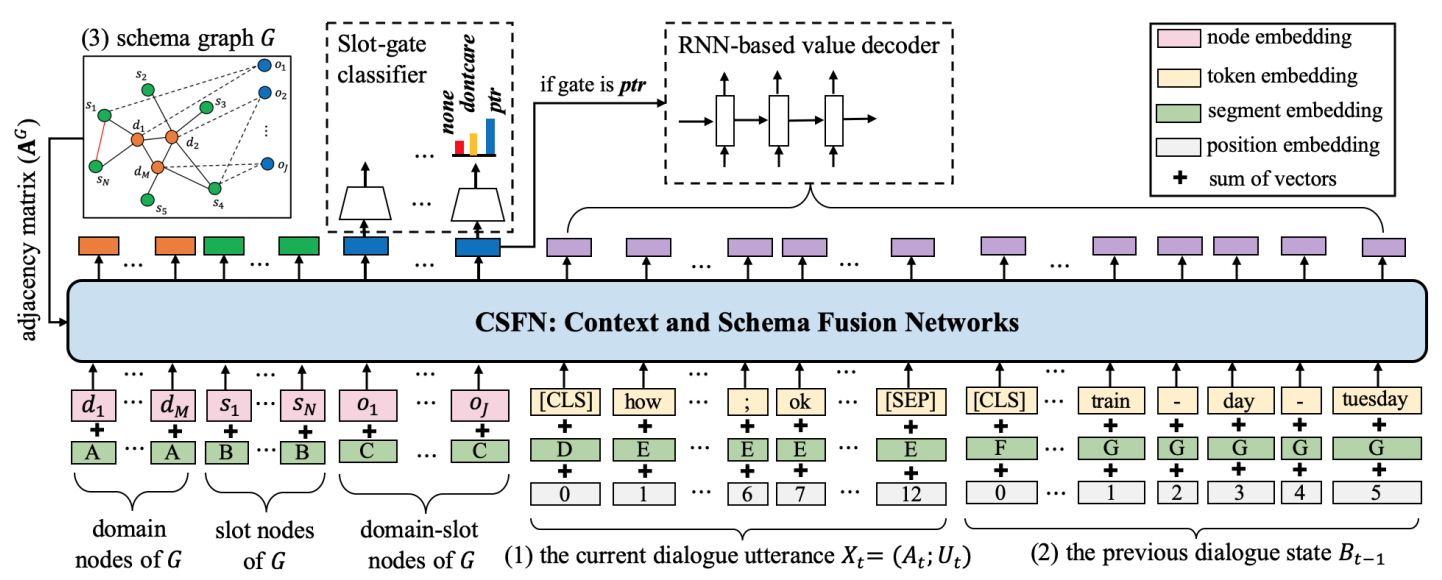

Figure 3: The overview of the proposed CSFN-DST. It takes the current dialogue utterance, the previous dialogue state and the schema graph as the input and predicts the current dialogue state. It consists of an embedding layer, context and schema fusion networks, a slot-gate classifier and an RNN-based value decoder.

\subsection{Input Embeddings}

Besides token and position embeddings for encoding literal information, segment embeddings are also exploited to discriminate different types of input tokens.

(1) Dialogue Utterance We denote the representation of the dialogue utterances at $t$-th turn as a joint sequence, $X_{t}=[\mathrm{CLS}] \oplus A_{t} \oplus ; \oplus U_{t} \oplus$ $[\mathrm{SEP}]$, where $[\mathrm{CLS}]$ and $[\mathrm{SEP}]$ are auxiliary tokens for separation, $\oplus$ is the operation of sequence concatenation. As [CLS] is designed to capture the sequence embedding, it has a different segment type with the other tokens. The input embeddings of $X_{t}$ are the sum of the token embeddings, the segmentation embeddings and the position embeddings (Vaswani et al., 2017), as shown in Fig. 3.

(2) Previous Dialogue State As mentioned before, a dialogue state is a set of domain-slot-value triplets with a mentioned value (not NONE). Therefore, we denote the previous dialogue state as $B_{t-1}=[\mathrm{CLS}] \oplus R_{t-1}^{1} \oplus \cdots \oplus R_{t-1}^{K}$, where $K$ is the number of triplets in $B_{t-1}$. Each triplet $d-s-v$ is denoted as a sub-sequence, i.e., $R=$ $d \oplus-\oplus s \oplus-\oplus v$. The domain and slot names are tokenized, e.g., price_range is replaced with "price range". The value is also represented as a token sequence. For the special value DONTCARE which means users do not care the value, it would be replaced with "dont care". The input embeddings of $B_{t-1}$ are the sum of the token, segmentation and position embeddings. Positions are re-enumerated for different triplets.

(3) Schema Graph As mentioned before, the schema graph $G$ is comprised of $M$ domain nodes, $N$ slot nodes and $J$ domain-slot nodes. These nodes are arranged as $G=d_{1} \oplus \cdots \oplus d_{M} \oplus s_{1} \oplus$ $\cdots \oplus s_{N} \oplus o_{1} \oplus \cdots \oplus o_{J}$. Each node embedding is initialized by averaging embeddings of tokens in the corresponding domain/slot/domain-slot. Positions embeddings are omitted in the graph. The edges of the graph are represented as an adjacency matrix $\mathbf{A}^{G}$ whose items are either one or zero, which would be used in the fusion network. To emphasize edges between different types of nodes can be different in the computation, we exploit node types to get segment embeddings.

\subsection{Context and Schema Fusion Network}

At this point, we have input representations $\mathrm{H}_{0}^{G} \in$ $\mathbb{R}^{|G| \times d_{m}}, \mathrm{H}_{0}^{X_{t}} \in \mathbb{R}^{\left|X_{t}\right| \times d_{m}}, \mathrm{H}_{0}^{B_{t-1}} \in \mathbb{R}^{\left|B_{t-1}\right| \times d_{m}}$, where |.| gets the token or node number. The context and schema fusion network (CSFN) is utilized to compute hidden states for tokens or nodes in $X_{t}$, $B_{t-1}$ and $G$ layer by layer. We then apply a stack of $L$ context- and schema-aware self-attention layers to get final hidden states, $\mathrm{H}_{L}^{G}, \mathrm{H}_{L}^{X_{t}}, \mathrm{H}_{L}^{B_{t-1}}$. The $i$-th layer $(0 \leq i<L)$ can be formulated as:

$\mathrm{H}_{i+1}^{G}, \mathrm{H}_{i+1}^{X_{t}}, \mathrm{H}_{i+1}^{B_{t-1}}=\operatorname{CSFNLayer}_{i}\left(\mathrm{H}_{i}^{G}, \mathrm{H}_{i}^{X_{t}}, \mathrm{H}_{i}^{B_{t-1}}\right)$

\subsubsection{Multi-head Attention}

Before describing the fusion network, we first introduce the multi-head attention (Vaswani et al., 2017) which is a basic module. The multi-head attention can be described as mapping a query and a set of key-value pairs to an output, where the query, keys, values, and output are all vectors. The output is computed as a weighted sum of the values, where the weight assigned to each value is 
computed by a compatibility function of the query with the corresponding key.

Consider a source sequence of vectors $Y=$

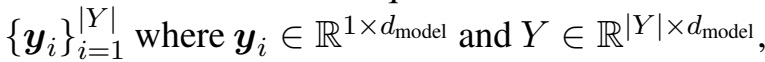
and a target sequence of vectors $Z=\left\{\boldsymbol{z}_{i}\right\}_{i=1}^{|Z|}$ where $\boldsymbol{z}_{i} \in \mathbb{R}^{1 \times d_{\text {model }}}$ and $Z \in \mathbb{R}^{|Z| \times d_{\text {model }} \text {. For }}$ each vector $\boldsymbol{y}_{i}$, we can compute an attention vector $c_{i}$ over $Z$ by using $H$ heads as follows:

$e_{i j}^{(h)}=\frac{\left(\boldsymbol{y}_{i} W_{Q}^{(h)}\right)\left(\boldsymbol{z}_{j} W_{K}^{(h)}\right)^{\top}}{\sqrt{d_{\text {model }} / H}} ; a_{i j}^{(h)}=\frac{\exp \left(e_{i j}^{(h)}\right)}{\sum_{l=1}^{|Z|} \exp \left(e_{i l}^{(h)}\right)}$

$\boldsymbol{c}_{i}^{(h)}=\sum_{j=1}^{|Z|} a_{i j}^{(h)}\left(\boldsymbol{z}_{j} W_{V}^{(h)}\right) ; \boldsymbol{c}_{i}=\operatorname{Concat}\left(\boldsymbol{c}_{i}^{(1)}, \cdots, \boldsymbol{c}_{i}^{(H)}\right) W_{O}$

where $1 \leq h \leq H, W_{O} \in \mathbb{R}^{d_{\text {model }} \times d_{\text {model }}}$, and $W_{Q}^{(h)}, W_{K}^{(\bar{h})}, W_{V}^{(\bar{h})} \in \mathbb{R}^{d_{\text {model }} \times\left(d_{\text {model }} / H\right)}$. We can compute $\boldsymbol{c}_{i}$ for every $\boldsymbol{y}_{i}$ and get a transformed matrix $C \in \mathbb{R}^{|Y| \times d_{\text {model }}}$. The entire process is denoted as a mapping MultiHead $_{\Theta}$ :

$$
C=\operatorname{MultiHead}_{\Theta}(Y, Z)
$$

Graph-based Multi-head Attention To apply the multi-head attention on a graph, the graph adjacency matrix $\mathbf{A} \in \mathbb{R}^{|Y| \times|Z|}$ is involved to mask nodes/tokens unrelated, where $\mathbf{A}_{i j} \in\{0,1\}$. Thus, $e_{i j}^{(h)}$ is changed as:

$$
e_{i j}^{(h)}= \begin{cases}\frac{\left(\boldsymbol{y}_{i} W_{Q}^{(h)}\right)\left(\boldsymbol{z}_{j} W_{K}^{(h)}\right)^{\top}}{\sqrt{d_{\text {model }} / H}}, & \text { if } \mathbf{A}_{i j}=1 \\ -\infty, & \text { otherwise }\end{cases}
$$

and Eqn. (1) is modified as:

$$
C=\operatorname{GraphMultiHead}_{\Theta}(Y, Z, \mathbf{A})
$$

Eqn. (1), can be treated as a special case of Eqn. (2) that the graph is fully connected, i.e., $\mathbf{A}=\mathbf{1}$.

\subsubsection{Context- and Schema-Aware Encoding}

Each layer of CSFN consists of internal and external attentions to incorporate different types of inputs. The hidden states of the schema graph $G$ at the $i$-the layer are updated as follows:

$$
\begin{aligned}
& \mathrm{I}_{\mathrm{GG}}=\text { GraphMultiHead }_{\Theta_{\mathrm{GG}}}\left(\mathrm{H}_{i}^{G}, \mathrm{H}_{i}^{G}, \mathbf{A}^{G}\right) \\
& \mathrm{E}_{\mathrm{GX}}=\text { MultiHead }_{\Theta_{\mathrm{GX}}}\left(\mathrm{H}_{i}^{G}, \mathrm{H}_{i}^{X_{t}}\right) \\
& \mathrm{E}_{\mathrm{GB}}=\text { MultiHead }_{\Theta_{\mathrm{GB}}}\left(\mathrm{H}_{i}^{G}, \mathrm{H}_{i}^{B_{t-1}}\right) \\
& \mathrm{C}_{G}=\operatorname{LayerNorm}\left(\mathrm{H}_{i}^{G}+\mathrm{I}_{\mathrm{GG}}+\mathrm{E}_{\mathrm{GX}}+\mathrm{E}_{\mathrm{GB}}\right) \\
& \mathrm{H}_{i+1}^{G}=\text { LayerNorm }\left(\mathrm{C}_{G}+\operatorname{FFN}\left(\mathrm{C}_{G}\right)\right)
\end{aligned}
$$

where $\mathbf{A}^{G}$ is the adjacency matrix of the schema graph and LayerNorm(.) is layer normalization function (Ba et al., 2016). $\operatorname{FFN}(x)$ is a feedforward network (FFN) function with two fullyconnected layer and an ReLU activation in between, i.e., $\operatorname{FFN}(x)=\max \left(0, x W_{1}+b_{1}\right) W_{2}+b_{2}$.

Similarly, more details about updating $\mathrm{H}_{i}^{X_{t}}, \mathrm{H}_{i}^{B_{t-1}}$ are described in Appendix A.

The context and schema-aware encoding can also be simply implemented as the original transformer (Vaswani et al., 2017) with graph-based multi-head attentions.

\subsection{State Prediction}

The goal of state prediction is to produce the next dialogue state $B_{t}$, which is formulated as two stages: 1) We first apply a slot-gate classifier for each domain-slot node. The classifier makes a decision among \{NONE, DONTCARE, PTR\}, where NONE denotes that a domain-slot pair is not mentioned at this turn, DONTCARE implies that the user can accept any values for this slot, and PTR represents that the slot should be processed with a value. 2) For domain-slot pairs tagged with PTR, we further introduced an RNN-based value decoder to generate token sequences of their values.

\subsubsection{Slot-gate Classification}

We utilize the final hidden vector of $j$-th domainslot node in $G$ for the slot-gate classification, and the probability for the $j$-th domain-slot pair at the $t$-th turn is calculated as:

$$
P_{t j}^{\text {gate }}=\operatorname{softmax}\left(\operatorname{FFN}\left(\mathrm{H}_{L, M+N+j}^{G}\right)\right)
$$

The loss for slot gate classification is

$$
\mathcal{L}_{\text {gate }}=-\sum_{t=1}^{T} \sum_{j=1}^{J} \log \left(P_{t j}^{\text {gate }} \cdot\left(y_{t j}^{\text {gate }}\right)^{\top}\right)
$$

where $y_{t j}^{\text {gate }}$ is the one-hot gate label for the $j$-th domain-slot pair at turn $t$.

\subsubsection{RNN-based Value Decoder}

After the slot-gate classification, there are $J^{\prime}$ domain-slot pairs tagged with PTR class which indicates the domain-slot should take a real value. They are denoted as $\mathbb{C}_{t}=\left\{j \mid \operatorname{argmax}\left(P_{t j}^{\text {gate }}\right)=\right.$ PTR $\}$, and $J^{\prime}=\left|\mathbb{C}_{t}\right|$.

We use Gated Recurrent Unit (GRU) (Cho et al., 2014) decoder like Wu et al. (2019) and the soft copy mechanism (See et al., 2017) to get the final output distribution $P_{t j}^{\mathrm{value}, k}$ over all candidate tokens at the $k$-th step. More details are illustrated in 
Appendix B. The loss function for value decoder is

$$
\mathcal{L}_{\text {value }}=-\sum_{t=1}^{T} \sum_{j \in \mathbb{C}_{t}} \sum_{k} \log \left(P_{t j}^{\text {value }, k} \cdot\left(y_{t j}^{\text {value }, k}\right)^{\top}\right)
$$

where $y_{t j}^{\text {value, } k}$ is the one-hot token label for the $j$-th domain-slot pair at $k$-th step.

During training process, the above modules can be jointly trained and optimized by the summations of different losses as:

$$
\mathcal{L}_{\text {total }}=\mathcal{L}_{\text {gate }}+\mathcal{L}_{\text {value }}
$$

\section{Experiment}

\subsection{Datasets}

We use MultiWOZ 2.0 (Budzianowski et al., 2018) and MultiWOZ 2.1 (Eric et al., 2019) to evaluate our approach. MultiWOZ 2.0 is a task-oriented dataset of human-human written conversations spanning over seven domains, consists of 10348 multi-turn dialogues. MultiWOZ 2.1 is a revised version of MultiWOZ 2.0, which is re-annotated with a different set of inter-annotators and also canonicalized entity names. According to the work of Eric et al. (2019), about $32 \%$ of the state annotations is corrected so that the effect of noise is counteracted.

Note that hospital and police are excluded since they appear in training set with a very low frequency, and they do not even appear in the test set. To this end, five domains (restaurant, train, hotel, taxi, attraction) are involved in the experiments with 17 distinct slots and 30 domain-slot pairs.

We follow similar data pre-processing procedures as Wu et al. (2019) on both MultiWOZ 2.0 and 2.1. ${ }^{2}$ The resulting corpus includes 8,438 multi-turn dialogues in training set with an average of 13.5 turns per dialogue. Data statistics of MultiWOZ 2.1 is shown in Table 1. The adjacency matrix $\mathbf{A}^{G}$ of MultiWOZ 2.0 and 2.1 datasets is shown in Figure 4 of Appendix, while domain-slot pairs are omitted due to space limitations.

\subsection{Experiment Settings}

We set the hidden size of CSFN, $d_{\text {model }}$, as 400 with 4 heads. Following $\mathrm{Wu}$ et al. (2019), the token embeddings with 400 dimensions are initialized by concatenating Glove embeddings (Pennington et al., 2014) and character embeddings

\footnotetext{
${ }^{2}$ https://github.com/budzianowski/ multiwoz
}

\begin{tabular}{|c|l|lll|}
\hline Domain & Slots & Train & Valid & Test \\
\hline Restaurant & $\begin{array}{l}\text { area, food, name, } \\
\text { price range, book day, } \\
\text { book people, book } \\
\text { time }\end{array}$ & & & \\
\hline Hotel & $\begin{array}{l}\text { area, internet, name, } \\
\text { parking, price range, } \\
\text { stars, type, book day, } \\
\text { book people, book } \\
\text { stay }\end{array}$ & & & \\
\hline Train & $\begin{array}{l}\text { arrive by, day, de- } \\
\text { parture, destination, } \\
\text { leave at, book people }\end{array}$ & & & \\
\hline Taxi & $\begin{array}{l}\text { arrive by, departure, } \\
\text { destination, leave at }\end{array}$ & 1654 & 207 & 195 \\
\hline Attraction & area, name, type & 2717 & 401 & 395 \\
\hline & Total & 8438 & 1000 & 1000 \\
\hline
\end{tabular}

Table 1: Data statistics of MultiWOZ2.1.

(Hashimoto et al., 2017). We do a grid search over $\{4,5,6,7,8\}$ for the layer number of CSFN on the validation set. We use a batch size of 32 . The DST model is trained using ADAM (Kingma and $\mathrm{Ba}, 2014$ ) with the learning rate of 1e-4. During training, we use the ground truth of the previous dialogue state and the ground truth value tokens. In the inference, the predicted dialogue state of the last turn is applied, and we use a greedy search strategy in the decoding process of the value decoder.

\subsection{Baseline Models}

We make a comparison with the following existing models, which are either predefined ontologybased DSTs or open-vocabulary based DSTs. Predefined ontology-based DSTs have the advantage of access to the known candidate set of each slot, while these approaches may not be applicable in the real scenario.

FJST (Eric et al., 2019): It exploits a bidirectional LSTM network to encode the dialog history and a separate FFN to predict the value for each slot.

HJST (Eric et al., 2019): It encodes the dialogue history using an LSTM like FJST, but utilizes a hierarchical network.

SUMBT (Lee et al., 2019): It exploits BERT (Devlin et al., 2018) as the encoder for the dialogue context and slot-value pairs. After that, it scores every candidate slot-value pair with the dialogue context by using a distance measure.

HyST (Goel et al., 2019): It is a hybrid approach based on hierarchical RNNs, which incorporates both a predefined ontology-based setting and an open-vocabulary setting.

DST-Reader (Gao et al., 2019): It models the DST 


\begin{tabular}{c|l|c||cc}
\hline & Models & BERT used & MultiWOZ 2.0 & MultiWOZ 2.1 \\
\hline \hline \multirow{5}{*}{$\begin{array}{c}\text { predefined } \\
\text { ontology }\end{array}$} & HJST (Eric et al., 2019)* & $\boldsymbol{x}$ & 38.40 & 35.55 \\
& FJST (Eric et al., 2019)* & $\boldsymbol{x}$ & 40.20 & 38.00 \\
& SUMBT (Lee et al., 2019) & $\checkmark$ & 42.40 & - \\
& HyST (Goel et al., 2019)* & $\boldsymbol{x}$ & 42.33 & 38.10 \\
& DS-DST (Zhang et al., 2019) & $\checkmark$ & - & 51.21 \\
& DST-Picklist (Zhang et al., 2019) & $\checkmark$ & - & 53.30 \\
& DSTQA (Zhou and Small, 2019) & $\boldsymbol{x}$ & $\mathbf{5 1 . 4 4}$ & 51.17 \\
& SST (Chen et al., 2020) & $\boldsymbol{x}$ & 51.17 & $\mathbf{5 5 . 2 3}$ \\
\hline \multirow{5}{*}{ open- } & DST-Span (Zhang et al., 2019) & $\checkmark$ & - & 40.39 \\
& DST-Reader (Gao et al., 2019)* & $\boldsymbol{x}$ & 39.41 & 36.40 \\
& TRADE (Wu et al., 2019)* & $\boldsymbol{x}$ & 48.60 & 45.60 \\
& COMER (Ren et al., 2019) & $\checkmark$ & 48.79 & - \\
& NADST (Le et al., 2020) & $\boldsymbol{x}$ & 50.52 & 49.04 \\
& SOM-DST (Kim et al., 2019) & $\checkmark$ & 51.72 & 53.01 \\
\cline { 2 - 5 } & CSFN-DST (ours) & $\boldsymbol{x}$ & 49.59 & 50.81 \\
& CSFN-DST + BERT (ours) & $\checkmark$ & 51.57 & 52.88 \\
& SOM-DST (our implementation) & $\checkmark$ & 51.66 & 52.85 \\
& SOM-DST + Schema Graph (ours) & $\checkmark$ & $\mathbf{5 2 . 2 3}$ & $\mathbf{5 3 . 1 9}$ \\
\hline
\end{tabular}

Table 2: Joint goal accuracy (\%) on the test set of MultiWOZ 2.0 and 2.1. * indicates a result borrowed from Eric et al. (2019). $\checkmark$ means that a BERT model (Devlin et al., 2018) with contextualized word embeddings is utilized.

from the perspective of text reading comprehensions, and get start and end positions of the corresponding text span in the dialogue context.

DST-Span (Zhang et al., 2019): It treats all domain-slot pairs as span-based slots like DSTReader, and applies a BERT as the encoder.

DST-Picklist (Zhang et al., 2019): It defines picklist-based slots for classification similarly to SUMBT and applies a pre-trained BERT for the encoder. It relies on a predefined ontology.

DS-DST (Zhang et al., 2019): Similar to HyST, it is a hybrid system of DS-Span and DS-Picklist.

DSTQA (Zhou and Small, 2019): It models multidomain DST as a question answering problem, and generates a question asking for the value of each domain-slot pair. It heavily relies on a predefined ontology, i.e., the candidate set for each slot is known, except for five time-related slots.

TRADE (Wu et al., 2019): It contains a slot gate module for slots classification and a pointer generator for dialogue state generation.

COMER (Ren et al., 2019): It uses a hierarchical decoder to generate the current dialogue state itself as the target sequence.

NADST (Le et al., 2020): It uses a nonautoregressive decoding scheme to generate the current dialogue state.

SST (Chen et al., 2020): It utilizes a graph attention matching network to fuse information from utterances and schema graphs, and a recurrent graph attention network to control state updating. However, it heavily relies on a predefined ontology.

SOM-DST (Kim et al., 2019): It uses a BERT to jointly encode the previous state, the previous and current dialogue utterances. An RNN-decoder is also applied to generate values for slots that need to be updated in the open-vocabulary setting.

\subsection{Main Results}

Joint goal accuracy is the evaluation metric in our experiments, which is represented as the ratio of turns whose predicted dialogue states are entirely consistent with the ground truth in the test set. Table 2 illustrates that the joint goal accuracy of CSFN-DST and other baselines on the test set of MultiWOZ 2.0 and MultiWOZ 2.1 datasets.

As shown in the table, our proposed CSFN-DST can outperform other models except for SOM-DST. By combining our schema graphs with SOM-DST, we can achieve state-of-the-art performances on both MultiWOZ 2.0 and 2.1 in the open-vocabulary setting. Additionally, our method using BERT (Bert-base-uncased) can obtain very competitive performance with the best systems in the predefined ontology-based setting. When a BERT is exploited, we initialize all parameters of CSFN with the BERT encoder's and initialize the token/position embeddings with the BERT's.

\subsection{Analysis}

In this subsection, we will conduct some ablation studies to figure out the potential factors for the improvement of our method. (Additional experiments and results are reported in Appendix C, case study is shown in Appendix D.) 


\begin{tabular}{|l|c|}
\hline Models & Joint Acc. (\%) \\
\hline CSFN-DST & 50.81 \\
\hline (-) Omit $\mathrm{H}_{L}^{B_{t-1}}$ in the decoder & 48.66 \\
(-) Omit $\mathrm{H}_{L}^{X_{t}}$ in the decoder & 48.45 \\
(+) The previous utterance $X_{t-1}$ & 50.75 \\
\hline
\end{tabular}

Table 3: Ablation studies for context information on MultiWOZ 2.1.

\begin{tabular}{|c|c|c|}
\hline \multirow{2}{*}{ Models } & \multicolumn{2}{|c|}{ BERT used } \\
\hline & $x$ & $\checkmark$ \\
\hline CSFN-DST & 50.81 & 52.88 \\
\hline (-) No schema graph, $\mathbf{A}^{G}=\mathbf{1}$ & 49.93 & 52.50 \\
\hline (-) No schema graph, $\mathbf{A}^{G}=\boldsymbol{I}$ & 49.52 & 52.46 \\
\hline (+) Ground truth of the previous state & 78.73 & 80.35 \\
\hline (+) Ground truth slot-gate classifi. & 77.31 & 80.66 \\
\hline (+) Ground truth value generation & 56.50 & 59.12 \\
\hline
\end{tabular}

Table 4: Joint goal accuracy(\%) of ablation studies on MultiWOZ 2.1.

\subsubsection{Effect of context information}

Context information consists of the previous dialogue state or the current dialogue utterance, which are definitely key for the encoder. It would be interesting to know whether the two kinds of context information are also essential for the RNN-based value decoder. As shown in Table 3, we choose to omit the top hidden states of the previous dialogue state $\left(\mathrm{H}_{L}^{B_{t-1}}\right)$ or the current utterance $\left(\mathrm{H}_{L}^{X_{t}}\right)$ in the RNN-based value decoder. The results show both of them are crucial for generating real values.

Do we need more context? Only the current dialogue utterance is utilized in our model, which would be more efficient than the previous methods involving all the preceding dialogue utterance. However, we want to ask whether the performance will be improved when more context is used. In Table 3, it shows that incorporating the previous dialogue utterance $X_{t-1}$ gives no improvement, which implies that jointly encoding the current utterance and the previous dialogue state is effective as well as efficient.

\subsubsection{Effect of the schema graph}

In CSFN-DST, the schema graph with domain-slot relations is exploited. To check the effectiveness of the schema graph used, we remove knowledgeaware domain-slot relations by replacing the adjacency matrix $\mathbf{A}^{G}$ as a fully connected one 1 or node-independent one $\boldsymbol{I}$. Results in Table 4 show that joint goal accuracies of models without the schema graph are decreased similarly when BERT is either used or not.

To reveal why the schema graph with domain-

\begin{tabular}{|c||c|c|c|c|c|}
\hline Models & Attr. & Hotel & Rest. & Taxi & Train \\
\hline \hline CSFN-DST & 64.78 & $\mathbf{4 6 . 2 9}$ & $\mathbf{6 4 . 6 4}$ & $\mathbf{4 7 . 3 5}$ & $\mathbf{6 9 . 7 9}$ \\
(-) No SG & $\mathbf{6 5 . 9 7}$ & 45.48 & 62.94 & 46.42 & 67.58 \\
\hline
\end{tabular}

Table 5: Domain-specific joint accuracy on MultiWOZ 2.1. SG means Schema Graph.

\begin{tabular}{|c|c||l|l|}
\hline Turn & Proportion (\%) & w/ SG & w/o SG \\
\hline \hline 1 & 13.6 & 89.39 & $88.19(-1.20)$ \\
2 & 13.6 & 73.87 & $72.87(-1.00)$ \\
3 & 13.4 & 58.69 & $57.78(-0.91)$ \\
4 & 12.8 & 51.96 & $50.80(-1.16)$ \\
5 & 11.9 & 41.01 & $39.63(-1.38)$ \\
6 & 10.7 & 34.51 & $35.15(+0.64)$ \\
7 & 9.1 & 27.91 & $29.55(+1.64)$ \\
8 & 6.3 & 24.73 & $23.23(-1.50)$ \\
9 & 4.0 & 20.55 & $19.18(-1.37)$ \\
10 & 2.3 & 16.37 & $12.28(-4.09)$ \\
11 & 1.3 & 12.63 & $8.42(-4.21)$ \\
12 & 0.6 & 12.77 & $8.51(-4.26)$ \\
$>12$ & 0.4 & 9.09 & $0.00(-9.09)$ \\
\hline all & 100 & 50.81 & 49.93 \\
\hline
\end{tabular}

Table 6: Joint accuracies over different dialogue turns on MultiWOZ 2.1. It shows the impact of using schema graph on our proposed CSFN-DST.

slot relations is essential for joint accuracy, we further make analysis on domain-specific and turnspecific results. As shown in Table 5, the schema graph can benefit almost all domains except for Attaction (Attr.). As illustrated in Table 1, the Attaction domain contains only three slots, which should be much simpler than the other domains. Therefore, we may say that the schema graph can help complicated domains.

The turn-specific results are shown in Table 6, where joint goal accuracies over different dialogue turns are calculated. From the table, we can see that data proportion of larger turn number becomes smaller while the larger turn number refers to more challenging conversation. From the results of the table, we can find the schema graph can make improvements over most dialogue turns.

\subsubsection{Oracle experiments}

The predicted dialogue state at the last turn is utilized in the inference stage, which is mismatched with the training stage. An oracle experiment is conducted to show the impact of training-inference mismatching, where ground truth of the previous dialogue state is fed into CSFN-DST. The results in Table 4 show that joint accuracy can be nearly $80 \%$ with ground truth of the previous dialogue state. Other oracle experiments with ground truth slot-gate classification and ground truth value generation are also conducted, as shown in Table 4. 


\subsubsection{Slot-gate classification}

We conduct experiments to evaluate our model performance on the slot-gate classification task. Table 7 shows $\mathrm{F} 1$ scores of the three slot gates, i.e., \{NONE, DONTCARE, PTR . It seems that the pretrained BERT model helps a lot in detecting slots of which the user doesn't care about values. The F1 score of DONTCARE is much lower than the others', which implies that detecting DONTCARE is a much challenging sub-task.

\begin{tabular}{c|c|c}
\hline Gate & CSFN-DST & CSFN-DST + BERT \\
\hline \hline NONE & 99.18 & 99.19 \\
\hline DONTCARE & 72.50 & 75.96 \\
\hline PTR & 97.66 & 98.05 \\
\hline
\end{tabular}

Table 7: Slot-gate F1 scores on MultiWOZ 2.1.

\subsection{Reproducibility}

We run our models on GeForce GTX $2080 \mathrm{Ti}$ Graphics Cards, and the average training time for each epoch and number of parameters in each model are provided in Table 8. If BERT is exploited, we accumulate the gradients with 4 steps for a minibatch of data samples (i.e., $32 / 4=8$ samples for each step), due to the limitation of GPU memory. As mentioned in Section 5.4, joint goal accuracy is the evaluation metric used in our experiments, and we follow the computing script provided in TRADE-DST ${ }^{3}$.

\begin{tabular}{|c|c|c|}
\hline Method & Time per Batch & \# Parameters \\
\hline CSFN-DST & $350 \mathrm{~ms}$ & $63 \mathrm{M}$ \\
CSFN-DST + BERT & $840 \mathrm{~ms}$ & $115 \mathrm{M}$ \\
SOM-DST + SG & $1160 \mathrm{~ms}$ & $115 \mathrm{M}$ \\
\hline
\end{tabular}

Table 8: Runtime and mode size of our methods.

\subsection{Discussion}

The main contributions of this work may focus on exploiting the schema graph with graph-based attention networks. Slot-relations are also utilized in DSTQA (Zhou and Small, 2019). However, DSTQA uses a dynamically-evolving knowledge graph for the dialogue context, and we use a static schema graph. We absorb the dialogue context by using the previous (predicted) dialogue state as another input. We believe that the two different usages of the slot relation graph can be complementary. Moreover, these two methods are different in

\footnotetext{
${ }^{3}$ https://github.com/jasonwu0731/ trade-dst
}

value prediction that DSTQA exploits a hybrid of value classifier and span prediction layer, which relies on a predefined ontology.

SOM-DST (Kim et al., 2019) is very similar to our proposed CSFN-DST with BERT. The main difference between SOM-DST and CSFN-DST is how to exploit the previous dialogue state. For the previous dialogue state, SOM-DST considers all domain-slot pairs and their values (if a domainslot pair contains an empty value, a special token NONE is used), while CSFN-DST only considers the domain-slot pairs with a non-empty value. Thus, SOM-DST knows which domain-slot pairs are empty and would like to be filled with a value. We think that it is the strength of SOM-DST. However, we choose to omit the domain-slot pairs with an empty value for a lower computation burden, which is proved in Table 8. As shown in the last two rows of Table 2, the schema graph can also improve SOM-DST, which achieves $52.23 \%$ and $53.19 \%$ joint accuracies on MultiWOZ 2.0 and 2.1, respectively. Appendix E shows how to exploit schema graph in SOM-DST.

\section{Conclusion and Future Work}

We introduce a multi-domain dialogue state tracker with context and schema fusion networks, which involves slot relations and learns deep representations for each domain-slot pair dependently. Slots from different domains and their relations are organized as a schema graph. Our approach outperforms strong baselines on both MultiWOZ 2.0 and 2.1 benchmarks. Ablation studies also show that the effectiveness of the schema graph.

It will be a future work to incorporate relations among dialogue states, utterances and domain schemata. To further mitigate the data sparsity problem of multi-domain DST, it would be also interesting to incorporate data augmentations (Zhao et al., 2019) and semi-supervised learnings (Lan et al., 2018; Cao et al., 2019).

\section{Acknowledgments}

We thank the anonymous reviewers for their thoughtful comments. This work has been supported by Shanghai Jiao Tong University Scientific and Technological Innovation Funds (YG2020YQ01) and No. SKLMCPTS2020003 Project. 


\section{References}

Jimmy Lei Ba, Jamie Ryan Kiros, and Geoffrey E Hinton. 2016. Layer normalization. arXiv preprint arXiv:1607.06450.

Dzmitry Bahdanau, Kyunghyun Cho, and Yoshua Bengio. 2014. Neural machine translation by jointly learning to align and translate. arXiv preprint arXiv:1409.0473.

Paweł Budzianowski, Tsung-Hsien Wen, Bo-Hsiang Tseng, Iñigo Casanueva, Stefan Ultes, Osman Ramadan, and Milica Gašić. 2018. MultiWOZ - a large-scale multi-domain wizard-of-Oz dataset for task-oriented dialogue modelling. In Proceedings of the 2018 Conference on Empirical Methods in Natural Language Processing, pages 5016-5026.

Ruisheng Cao, Su Zhu, Chen Liu, Jieyu Li, and Kai Yu. 2019. Semantic parsing with dual learning. In Proceedings of the 57th Annual Meeting of the Association for Computational Linguistics, pages 51-64.

Lu Chen, Zhi Chen, Bowen Tan, Sishan Long, Milica Gasic, and Kai Yu. 2019. AgentGraph: Towards universal dialogue management with structured deep reinforcement learning. IEEE/ACM Transactions on Audio, Speech, and Language Processing, 27(9):1378-1391.

Lu Chen, Boer Lv, Chi Wang, Su Zhu, Bowen Tan, and Kai Yu. 2020. Schema-guided multi-domain dialogue state tracking with graph attention neural networks. In $A A A I$, pages 7521-7528.

Lu Chen, Bowen Tan, Sishan Long, and Kai Yu. 2018. Structured dialogue policy with graph neural networks. In Proceedings of the 27th International Conference on Computational Linguistics, pages 1257-1268.

Kyunghyun Cho, Bart Van Merriënboer, Dzmitry Bahdanau, and Yoshua Bengio. 2014. On the properties of neural machine translation: Encoder-decoder approaches. arXiv preprint arXiv:1409.1259.

Jacob Devlin, Ming-Wei Chang, Kenton Lee, and Kristina Toutanova. 2018. BERT: Pre-training of deep bidirectional transformers for language understanding. arXiv preprint arXiv:1810.04805.

Mihail Eric, Rahul Goel, Shachi Paul, Abhishek Sethi, Sanchit Agarwal, Shuyag Gao, and Dilek HakkaniTur. 2019. MultiWOZ 2.1: Multi-domain dialogue state corrections and state tracking baselines. arXiv preprint arXiv:1907.01669.

Shuyang Gao, Abhishek Sethi, Sanchit Agarwal, Tagyoung Chung, and Dilek Hakkani-Tur. 2019. Dialog state tracking: A neural reading comprehension approach. In Proceedings of the 20th Annual SIGdial Meeting on Discourse and Dialogue, pages 264 273.
Rahul Goel, Shachi Paul, and Dilek Hakkani-Tür. 2019. HyST: A hybrid approach for flexible and accurate dialogue state tracking. arXiv preprint arXiv:1907.00883.

Kazuma Hashimoto, Caiming Xiong, Yoshimasa Tsuruoka, and Richard Socher. 2017. A joint many-task model: Growing a neural network for multiple NLP tasks. In Proceedings of the 2017 Conference on Empirical Methods in Natural Language Processing, pages 1923-1933.

Matthew Henderson, Blaise Thomson, and Jason D Williams. 2014a. The second dialog state tracking challenge. In Proceedings of Annual SIGdial Meeting on Discourse and Dialogue, pages 263-272.

Matthew Henderson, Blaise Thomson, and Steve Young. 2014b. Robust dialog state tracking using delexicalised recurrent neural networks and unsupervised adaptation. In Proceedings of IEEE Spoken Language Technology Workshop (SLT).

Matthew Henderson, Blaise Thomson, and Steve Young. 2014c. Word-based dialog state tracking with recurrent neural networks. In Proceedings of Annual SIGdial Meeting on Discourse and Dialogue, pages 292-299.

Mettew Henderson, Blaise Thomson, and Jason D. Williams. 2014d. The third dialog state tracking challenge. In Proceedings of IEEE Spoken Language Technology Workshop (SLT).

Sungdong Kim, Sohee Yang, Gyuwan Kim, and SangWoo Lee. 2019. Efficient dialogue state tracking by selectively overwriting memory. arXiv preprint arXiv:1911.03906.

Diederik P Kingma and Jimmy Ba. 2014. Adam: A method for stochastic optimization. arXiv preprint arXiv:1412.6980.

Ouyu Lan, Su Zhu, and Kai Yu. 2018. Semi-supervised training using adversarial multi-task learning for spoken language understanding. In 2018 IEEE International Conference on Acoustics, Speech and Signal Processing (ICASSP), pages 6049-6053. IEEE.

Hung Le, Richard Socher, and Steven C.H. Hoi. 2020. Non-autoregressive dialog state tracking. In International Conference on Learning Representations.

Hwaran Lee, Jinsik Lee, and Tae-Yoon Kim. 2019. SUMBT: Slot-utterance matching for universal and scalable belief tracking. In Proceedings of the 57th Annual Meeting of the Association for Computational Linguistics, pages 5478-5483.

Diego Marcheggiani, Joost Bastings, and Ivan Titov. 2018. Exploiting semantics in neural machine translation with graph convolutional networks. arXiv preprint arXiv:1804.08313. 
Nikola Mrkšić, Diarmuid Ó Séaghdha, Tsung-Hsien Wen, Blaise Thomson, and Steve Young. 2017. Neural belief tracker: Data-driven dialogue state tracking. In Proceedings of the 55th Annual Meeting of the Association for Computational Linguistics, pages $1777-1788$.

Jeffrey Pennington, Richard Socher, and Christopher Manning. 2014. Glove: Global vectors for word representation. In Proceedings of the 2014 Conference on Empirical Methods in Natural Language Processing (EMNLP), pages 1532-1543.

Osman Ramadan, Paweł Budzianowski, and Milica Gasic. 2018. Large-scale multi-domain belief tracking with knowledge sharing. In Proceedings of the 56th Annual Meeting of the Association for Computational Linguistics, pages 432-437.

Liliang Ren, Jianmo Ni, and Julian McAuley. 2019. Scalable and accurate dialogue state tracking via hierarchical sequence generation. In Proceedings of the 2019 Conference on Empirical Methods in Natural Language Processing and the 9th International Joint Conference on Natural Language Processing (EMNLP-IJCNLP), pages 1876-1885.

Liliang Ren, Kaige Xie, Lu Chen, and Kai Yu. 2018. Towards universal dialogue state tracking. In Proceedings of the 2018 Conferenceon Empirical Methods in Natural Language Processing (EMNLP), pages 2780-2786.

Franco Scarselli, Marco Gori, Ah Chung Tsoi, Markus Hagenbuchner, and Gabriele Monfardini. 2009. The graph neural network model. IEEE Transactions on Neural Networks, 20(1):61-80.

Abigail See, Peter J Liu, and Christopher D Manning. 2017. Get to the point: Summarization with pointer-generator networks. arXiv preprint arXiv:1704.04368.

Kai Sun, Lu Chen, Su Zhu, and Kai Yu. 2014a. A generalized rule based tracker for dialogue state tracking. In Proceedings of IEEE Spoken Language Technology Workshop (SLT).

Kai Sun, Lu Chen, Su Zhu, and Kai Yu. 2014b. The SJTU system for dialog state tracking challenge 2. In Proceedings of the 15th Annual Meeting of the Special Interest Group on Discourse and Dialogue (SIGDIAL), pages 318-326.

Ashish Vaswani, Noam Shazeer, Niki Parmar, Jakob Uszkoreit, Llion Jones, Aidan N Gomez, Łukasz Kaiser, and Illia Polosukhin. 2017. Attention is all you need. In Advances in neural information processing systems.

Petar Veličković, Guillem Cucurull, Arantxa Casanova, Adriana Romero, Pietro Liò, and Yoshua Bengio. 2018. Graph attention networks. In Proceedings of International Conference on Learning Representations.
Jason Williams, Antoine Raux, Deepak Ramachandran, and Alan Black. 2013. The dialog state tracking challenge. In Proceedings of Annual SIGdial Meeting on Discourse and Dialogue, pages 404-413.

Jason D Williams. 2012. A belief tracking challenge task for spoken dialog systems. In NAACL-HLT Workshop on Future Directions and Needs in the Spoken Dialog Community: Tools and Data, pages 23-24.

Chien-Sheng Wu, Andrea Madotto, Ehsan HosseiniAsl, Caiming Xiong, Richard Socher, and Pascale Fung. 2019. Transferable multi-domain state generator for task-oriented dialogue systems. In Proceedings of the 57th Annual Meeting of the Association for Computational Linguistics.

Puyang Xu and Qi Hu. 2018. An end-to-end approach for handling unknown slot values in dialogue state tracking. In Proceedings of the 56th Annual Meeting of the Association for Computational Linguistics.

Liang Yao, Chengsheng Mao, and Yuan Luo. 2019. Graph convolutional networks for text classification. In Proceedings of the AAAI Conference on Artificial Intelligence, pages 7370-7377.

Steve Young, Milica Gašić, Blaise Thomson, and Jason D Williams. 2013. Pomdp-based statistical spoken dialog systems: A review. Proceedings of the IEEE, 101(5):1160-1179.

Kai Yu, Kai Sun, Lu Chen, and Su Zhu. 2015. Constrained markov bayesian polynomial for efficient dialogue state tracking. IEEE/ACM Transactions on Audio, Speech, and Language Processing, 23(12):2177-2188.

Jian-Guo Zhang, Kazuma Hashimoto, Chien-Sheng Wu, Yao Wan, Philip S Yu, Richard Socher, and Caiming Xiong. 2019. Find or classify? dual strategy for slot-value predictions on multi-domain dialog state tracking. arXiv preprint arXiv:1910.03544.

Zijian Zhao, Su Zhu, and Kai Yu. 2019. Data augmentation with atomic templates for spoken language understanding. In Proceedings of the 2019 Conference on Empirical Methods in Natural Language Processing and the 9th International Joint Conference on Natural Language Processing (EMNLPIJCNLP), pages 3628-3634.

Victor Zhong, Caiming Xiong, and Richard Socher. 2018. Global-locally self-attentive encoder for dialogue state tracking. In Proceedings of the 56th Annual Meeting of the Association for Computational Linguistics, pages 1458-1467.

Li Zhou and Kevin Small. 2019. Multi-domain dialogue state tracking as dynamic knowledge graph enhanced question answering. arXiv preprint arXiv:1911.06192. 


\section{A Context- and Schema-Aware Encoding}

Besides the hidden states $\mathrm{H}_{i}^{G}$ of the schema graph $G$, we show the details of updating $\mathrm{H}_{i}^{X_{t}}, \mathrm{H}_{i}^{B_{t-1}}$ in the $i$-th layer of CSFN:

$$
\mathrm{H}_{i+1}^{G}, \mathrm{H}_{i+1}^{X_{t}}, \mathrm{H}_{i+1}^{B_{t-1}}=\operatorname{CSFNLayer}_{i}\left(\mathrm{H}_{i}^{G}, \mathrm{H}_{i}^{X_{t}}, \mathrm{H}_{i}^{B_{t-1}}\right)
$$

The hidden states of the dialogue utterance $X_{t}$ at the $i$-the layer are updated as follows:

$$
\begin{aligned}
\mathrm{I}_{\mathrm{XX}} & =\text { MultiHead }_{\Theta_{\mathrm{XX}}}\left(\mathrm{H}_{i}^{X_{t}}, \mathrm{H}_{i}^{X_{t}}\right) \\
\mathrm{E}_{\mathrm{XB}} & =\text { MultiHead }_{\Theta_{\mathrm{XB}}}\left(\mathrm{H}_{i}^{X_{t}}, \mathrm{H}_{i}^{B_{t-1}}\right) \\
\mathrm{E}_{\mathrm{XG}} & =\text { MultiHead }_{\Theta_{\mathrm{XG}}}\left(\mathrm{H}_{i}^{X_{t}}, \mathrm{H}_{i}^{G}\right) \\
\mathrm{C}_{X} & =\operatorname{LayerNorm}_{(}\left(\mathrm{H}_{i}^{X_{t}}+\mathrm{I}_{\mathrm{XX}}+\mathrm{E}_{\mathrm{XB}}+\mathrm{E}_{\mathrm{XG}}\right) \\
\mathrm{H}_{i+1}^{X_{t}} & =\text { LayerNorm }\left(\mathrm{C}_{X}+\mathrm{FFN}\left(\mathrm{C}_{X}\right)\right)
\end{aligned}
$$

where $\mathrm{I}_{\mathrm{XX}}$ contains internal attention vectors, $\mathrm{E}_{\mathrm{XB}}$ and $E_{X G}$ are external attention vectors.

The hidden states of the previous dialogue state $B_{t-1}$ at the $i$-the layer are updated as follows:

$$
\begin{aligned}
\mathrm{I}_{\mathrm{BB}} & =\text { GraphMultiHead }_{\Theta_{\mathrm{BB}}}\left(\mathrm{H}_{i}^{B_{t-1}}, \mathrm{H}_{i}^{B_{t-1}}, \mathbf{A}^{B_{t-1}}\right) \\
\mathrm{E}_{\mathrm{BX}} & =\text { MultiHead }_{\Theta_{\mathrm{BX}}}\left(\mathrm{H}_{i}^{B_{t-1}}, \mathrm{H}_{i}^{X_{t}}\right) \\
\mathrm{E}_{\mathrm{BG}} & =\text { MultiHead }_{\Theta_{\mathrm{BG}}}\left(\mathrm{H}_{i}^{B_{t-1}}, \mathrm{H}_{i}^{G}\right) \\
\mathrm{C}_{B} & =\operatorname{LayerNorm}\left(\mathrm{H}_{i}^{B_{t-1}}+\mathrm{I}_{\mathrm{BB}}+\mathrm{E}_{\mathrm{BX}}+\mathrm{E}_{\mathrm{BG}}\right) \\
\mathrm{H}_{i+1}^{B_{t-1}} & =\text { LayerNorm }\left(\mathrm{C}_{B}+\mathrm{FFN}\left(\mathrm{C}_{B}\right)\right)
\end{aligned}
$$

where $\mathbf{A}^{B_{t-1}}$ is the adjacency matrix of the previous dialogue state. The adjacency matrix indicates that each triplets in $B_{t-1}$ is separated, while tokens in a same triplet are connected with each other. The [CLS ] token is connected with all triplets, serving as a transit node.

\section{B RNN-based Value Decoder}

After the slot-gate classification, there are $J^{\prime}$ domain-slot pairs tagged with PTR class which indicates the domain-slot should take a real value. They are denoted as $\mathbb{C}_{t}=\left\{j \mid \operatorname{argmax}\left(P_{t j}^{\text {gate }}\right)=\right.$ $\operatorname{PTR}\}$, and $J^{\prime}=\left|\mathbb{C}_{t}\right|$.

We use Gated Recurrent Unit (GRU) (Cho et al., 2014) decoder like Wu et al. (2019) and See et al. (2017). The hidden state $g_{t j}^{k} \in \mathbb{R}^{1 \times d_{\text {model }}}$ is recursively updated by taking a word embedding $e_{t j}^{k}$ as the input until [EOS] token is generated:

$$
g_{t j}^{k}=\operatorname{GRU}\left(g_{t j}^{k-1}, e_{t j}^{k}\right)
$$

GRU is initialized with

$$
g_{t j}^{0}=\mathrm{H}_{L, 0}^{X_{t}}+\mathrm{H}_{L, 0}^{B_{t-1}}
$$

and $e_{t j}^{0}=\mathrm{H}_{L, M+N+j}^{G}$.

The value generator transforms the hidden state to the probability distribution over the token vocabulary at the $k$-th step, which consists of two parts: 1) distribution over all input tokens, 2) distribution over the input vocabulary. The first part is computed as

$$
P_{t j}^{\mathrm{ctx}, k}=\operatorname{softmax}\left(\operatorname{ATT}\left(g_{t j}^{k},\left[\mathrm{H}_{L}^{X_{t}} ; \mathrm{H}_{L}^{B_{t-1}}\right]\right)\right)
$$

where $P_{t j}^{\mathrm{ctx}, k} \in \mathbb{R}^{1 \times\left(\left|X_{t}\right|+\left|B_{t-1}\right|\right)}$, and $\operatorname{ATT}(.,$.$) is a$ function to get attention weights (Bahdanau et al., 2014) with more details shown in Appendix B.1. The second part is calculated as

$$
\begin{aligned}
c_{t j}^{k} & =P_{t j}^{\mathrm{ctx}, k}\left[\mathrm{H}_{L}^{X_{t}} ; \mathrm{H}_{L}^{B_{t-1}}\right] \\
P_{t j}^{\mathrm{vocab}, k} & =\operatorname{softmax}\left(\left[g_{t j}^{k} ; c_{t j}^{k}\right] W_{\text {proj }} E^{\top}\right)
\end{aligned}
$$

where $P_{t j}^{\text {vocab }, k} \in \mathbb{R}^{1 \times d_{\text {vocab }}}, c_{t j}^{k} \in \mathbb{R}^{1 \times d_{\text {model }}}$ is a context vector, $W_{\text {proj }} \in \mathbb{R}^{2 d_{\text {model }} \times d_{\text {model }}}$ is a trainable parameter, and $E \in \mathbb{R}^{d_{\text {vocab }} \times d_{\text {model }}}$ is the token embedding matrix shared across the encoder and the decoder.

We use the soft copy mechanism (See et al., 2017) to get the final output distribution over all candidate tokens:

$$
\begin{aligned}
P_{t j}^{\text {value }, k} & =p_{\text {gen }} P_{t j}^{\text {vocab }, k}+\left(1-p_{\text {gen }}\right) P_{t j}^{\mathrm{ctx}, k} \\
p_{\text {gen }} & =\operatorname{sigmoid}\left(\left[g_{t j}^{k} ; e_{t j}^{k} ; c_{t j}^{k}\right] W_{\text {gen }}\right)
\end{aligned}
$$

where $W_{\text {gen }} \in \mathbb{R}^{3 d_{\text {model }} \times 1}$ is a trainable parameter.

The loss function for value decoder is

$\mathcal{L}_{\text {value }}=-\sum_{t=1}^{T} \sum_{j \in \mathbb{C}_{t}} \sum_{k} \log \left(P_{t j}^{\text {value }, k} \cdot\left(y_{t j}^{\text {value }, k}\right)^{\top}\right)$

where $y_{t j}^{\text {value, } k}$ is the one-hot token label for the $j$-th domain-slot pair at $k$-th step.

\section{B.1 Attention Weights}

For attention mechanism for computing $P_{t j}^{\mathrm{ctx}, k}$ in the RNN-based value decoder, we follow Bahdanau et al. (2014) and define the $\operatorname{ATT}(.,$.$) function as$

$$
\begin{aligned}
u_{i} & =\tanh \left(\mathbf{x} \mathbf{W}_{1}^{\text {att }}+\mathbf{h}_{i} \mathbf{W}_{2}^{\text {att }}+\mathbf{b}^{\text {att }}\right) \mathbf{v}^{\top} \\
a_{i} & =\frac{\exp \left(u_{i}\right)}{\sum_{j=1}^{S} \exp \left(u_{j}\right)} \\
\mathbf{a} & =\left\{a_{1}, \cdots, a_{S}\right\}=\operatorname{ATT}(\mathbf{x}, \mathrm{H})
\end{aligned}
$$


where $\mathbf{x} \in \mathbb{R}^{1 \times d}, \mathrm{H} \in \mathbb{R}^{S \times d}, \mathbf{W}_{1}^{\text {att }} \in \mathbb{R}^{d \times d}, \mathbf{W}_{2}^{\text {att }} \in$ $\mathbb{R}^{d \times d}, \mathbf{b}^{\text {att }} \in \mathbb{R}^{1 \times d}, \mathbf{v} \in \mathbb{R}^{1 \times d}$, and $\mathbf{h}_{i}$ is the $i$-th row vector of $\mathrm{H}$. Therefore, $\operatorname{ATT}(\mathbf{x}, \mathrm{H})$ returns an attention distribution of $\mathbf{x}$ over $\mathrm{H}$.

\section{Additional Results}

Domain-specific Results Domain-specific accuracy is the joint goal accuracy measured on a subset of the predicted dialogue state, which only contains the slots belong to a domain. From the results of Table 9, we can find BERT can make improvements on all domains, and especially the improvement on Taxi domain is the largest.

\begin{tabular}{|c||c|c|}
\hline Domain & CSFN-DST & CSFN-DST + BERT \\
\hline \hline Attraction & 64.78 & 67.82 \\
\hline Hotel & 46.29 & 48.80 \\
\hline Restaurant & 64.64 & 65.23 \\
\hline Taxi & 47.35 & 53.58 \\
\hline Train & 69.79 & 70.91 \\
\hline
\end{tabular}

Table 9: Domain-specific joint accuracy on MultiWOZ 2.1 .

Slot-specific Results Slot-specific F1 score is measured for predicting slot-value pairs of the corresponding slot. Table 10 shows slot-specific F1 scores of CSFN-DST without the schema graph, CSFN-DST and CSFN-DST with BERT on the test set of MultiWOZ 2.1.

\section{Case Study}

We also conduct case study on the test set of MultiWOZ 2.1, and four cases are shown in Table 11. From the first three cases, we can see the schema graph can copy values from related slots in the memory (i.e., the previous dialogue state). In the case $\mathrm{C} 1$, the model makes the accurate reference of the phrase "whole group" through the context, and the value of restaurant-book_people is copied as the value of train-book people. We can also see a failed case (C4). It is too complicated to inference the departure and destination by a word "commute".

\section{E SOM-DST with Schema Graph}

For SOM-DST (Kim et al., 2019), the input tokens to the state operation predictor are the concatenation of the previous turn dialog utterances, the current turn dialog utterances, and the previous turn dialog state:

$$
X_{t}=[\mathrm{CLS}] \oplus D_{t-1} \oplus D_{t} \oplus B_{t-1},
$$

where $D_{t-1}$ and $D_{t}$ are the last and current utterances, respectively. The dialogue state $B_{t}$ is denoted as $B_{t}=B_{t}^{1} \oplus \ldots \oplus B_{t}^{J}$, where $B_{t}^{j}=$ $[\mathrm{SLOT}]^{j} \oplus S^{j} \oplus-\oplus V_{t}^{j}$ is the representation of the $j$-th slot-value pair. To incorporate the schema graph, we exploit the special token $[\mathrm{SLOT}]^{j}$ to replace the domain-slot node $o_{j}$ in the schema graph $(j=1, \cdots, J)$. Then, domain and slot nodes $G^{\prime}=d_{1} \oplus \cdots \oplus d_{M} \oplus s_{1} \oplus \cdots \oplus s_{N}$ are concatenated into $X_{t}$, i.e.,

$$
X_{t}=[\mathrm{CLS}] \oplus D_{t-1} \oplus D_{t} \oplus B_{t-1} \oplus G^{\prime},
$$

where the relations among domain, slot and domain-slot nodes are also considered in attention masks of BERT. 


8
8

Figure 4: Adjacency matrix $\mathbf{A}^{G}$ of MultiWOZ 2.0 and 2.1 datasets. It contains only domain and slot nodes, while domain-slot paris are omitted due to space limitations. The first five items are domains ("attraction, hotel, restaurant, taxi, train"), and the rest are slots. 


\begin{tabular}{|c||c|c|c|}
\hline Domain-slot & CSFN-DST (no SG) & CSFN-DST & CSFN-DST + BERT \\
\hline \hline attraction-area & 91.67 & 91.92 & $\mathbf{9 2 . 8 1}$ \\
attraction-name & 78.28 & 77.77 & $\mathbf{7 9 . 5 5}$ \\
attraction-type & 90.95 & 90.89 & $\mathbf{9 1 . 9 7}$ \\
hotel-area & 84.59 & 84.21 & $\mathbf{8 4 . 8 6}$ \\
hotel-book_day & 97.16 & $\mathbf{9 7 . 7 9}$ & 97.03 \\
hotel-book_people & 95.43 & 96.14 & $\mathbf{9 7 . 3 5}$ \\
hotel-book_stay & 96.04 & $\mathbf{9 7 . 0 0}$ & 96.98 \\
hotel-internet & 86.79 & $\mathbf{8 9 . 9 8}$ & 86.58 \\
hotel-name & 82.97 & 83.11 & $\mathbf{8 4 . 6 1}$ \\
hotel-parking & 86.68 & $\mathbf{8 7 . 6 6}$ & 86.07 \\
hotel-price_range & 89.09 & 90.10 & $\mathbf{9 2 . 5 6}$ \\
hotel-stars & 91.51 & $\mathbf{9 3 . 4 9}$ & 93.34 \\
hotel-type & 77.58 & 77.87 & $\mathbf{8 2 . 1 2}$ \\
restaurant-area & 93.73 & $\mathbf{9 4 . 2 7}$ & 94.09 \\
restaurant-book_day & $\mathbf{9 7 . 7 5}$ & 97.66 & 97.42 \\
restaurant-book_people & 96.79 & 96.67 & $\mathbf{9 7 . 8 4}$ \\
restaurant-book_time & 92.43 & 91.60 & $\mathbf{9 4 . 2 9}$ \\
restaurant-food & $\mathbf{9 4 . 9 0}$ & 94.18 & 94.48 \\
restaurant-name & 80.72 & $\mathbf{8 1 . 3 9}$ & 80.59 \\
restaurant-price_range & 93.47 & 94.28 & $\mathbf{9 4 . 4 9}$ \\
taxi-arrive_by & 78.81 & 81.09 & $\mathbf{8 6 . 0 8}$ \\
taxi-departure & 73.15 & 71.39 & $\mathbf{7 5 . 1 5}$ \\
taxi-destination & 73.79 & 78.06 & $\mathbf{7 9 . 8 3}$ \\
taxi-leave_at & 77.29 & 80.13 & $\mathbf{8 8 . 0 6}$ \\
train-arrive_by & 86.43 & 87.56 & $\mathbf{8 8 . 7 7}$ \\
train-book_people & 89.59 & 91.41 & $\mathbf{9 2 . 3 3}$ \\
train-day & 98.44 & 98.41 & $\mathbf{9 8 . 5 2}$ \\
train-departure & 95.91 & $\mathbf{9 6 . 5 2}$ & 96.22 \\
train-destination & $\mathbf{9 7 . 0 8}$ & 97.06 & 96.13 \\
train-leave_at & 69.97 & 70.97 & $\mathbf{7 4 . 5 0}$ \\
\hline Joint Acc. overall & 49.93 & 50.81 & 52.88 \\
\hline
\end{tabular}

Table 10: Slot-specific F1 scores on MultiWOZ 2.1. SG means Schema Graph. The results in bold black are the best slot F1 scores. 


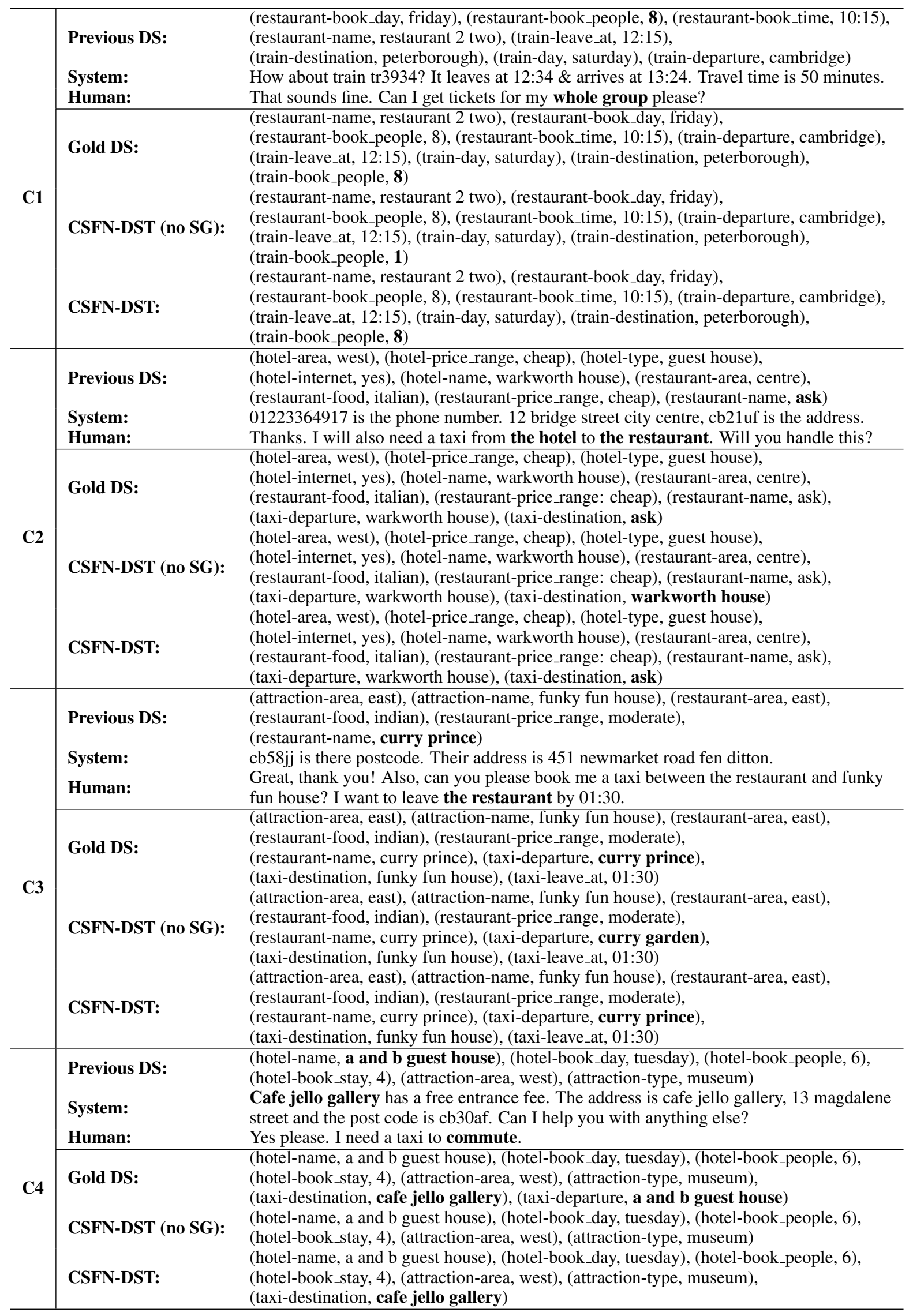

Table 11: Four cases on the test set of MultiWOZ 2.1. DS means Dialogue State, and SG means Schema Graph. 